\title{
Correction to: Clinical and demographic characteristics of primary progressive multiple sclerosis in Argentina: Argentinean registry cohort study (RelevarEM)
}

\begin{abstract}
Ricardo Alonso ${ }^{1,2,3}$ (1) Cecilia Quarracino ${ }^{3,4} \cdot$ Bárbara Eizaguirre $^{1,3} \cdot$ Leila Cohen $^{1,3}$ - Berenice Silva ${ }^{1,3}$. Cecilia Pita ${ }^{1,3}$. Juan Ignacio Rojas ${ }^{3,5}$. Agustín Pappolla ${ }^{3,5}$. Jimena Miguez ${ }^{3,5}$. Liliana Patrucco ${ }^{3,5}$. Edgardo Cristiano $^{3,5}$. Carlos Vrech ${ }^{3,6}$. Gabriel Volman ${ }^{3,7} \cdot$ Emanuel Silva $^{3,8}$. Andres Barboza ${ }^{3,9} \cdot$ Norma Deri $^{3,10}$. Verónica Tkachuk ${ }^{3,11}$. Gisela Zanga ${ }^{3,12}$. Dario Tavolini, 13,14 María Laura Saladino $^{3,15}$. Geraldine Luetic ${ }^{3,16}$. María Laura Menichini ${ }^{3,16}$. Edgar Carnero Contentti ${ }^{3,17} \cdot$ Pablo López $^{3,17}$. Judith Steinberg ${ }^{3,18}$. Pablo Divi ${ }^{3,19}$. Javier Hryb ${ }^{3,20}$. Amelia Alves Pinheiro ${ }^{3,21}$. Adriana Carra ${ }^{3,17,22}$ - María Eugenia Balbuena ${ }^{3,11}$ • Luciana Lázaro 2,3 . Nora Fernández Liguori ${ }^{2,3}$. Orlando Garcea ${ }^{1,3}$. on behalf of RelevarEM investigators
\end{abstract}

Published online: 24 September 2021

(c) Fondazione Società Italiana di Neurologia 2021

Correction to: Neurological Sciences (2020) 41:3329-3335 https://doi.org/10.1007/s10072-020-04680-3

The above article was published with inverted given and family names. This has been correctly presented above.

The Original article has been corrected.

The original article can be found online at https://doi.org/10.1007/ s10072-020-04680-3.

Ricardo Alonso

ricardoalonsohrm@gmail.com

1 Centro Universitario de Esclerosis Múltiple, Hospital Dr. J. M. Ramos Mejía, Facultad de Medicina-UBA, Urquiza 609, C1221ADC Buenos Aires City, Argentina

2 Sanatorio Güemes, Buenos Aires, Argentina

3 Sinapsis, Centro Neurológico, La Pampa, Argentina

4 Departamento de Neurología, Instituto de Investigaciones Médicas Alfredo Lanari, Buenos Aires, Argentina

5 Centro de Esclerosis Múltiple de Buenos Aires, Hospital Italiano de Buenos Aires, Buenos Aires, Argentina

6 Departamento de Enfermedades Desmielinizantes, Sanatorio Allende, Córdoba, Argentina

7 Departamento de Neurología, Hospital Presidente Perón de Avellaneda, Buenos Aires, Argentina

8 Predigma Centro de Medicina Preventiva, Posadas, Misiones, Argentina

9 Departamento de Neurología, Hospital Central de Mendoza, Mendoza, Argentina

10 Centro de Investigaciones Diabaid, Buenos Aires, Argentina
Publisher's note Springer Nature remains neutral with regard to jurisdictional claims in published maps and institutional affiliations.
11 Sección de Neuroinmunología y Enfermedades Desmielinizantes, Servicio de Neurología, Hospital de Clínicas José de San Martin, Buenos Aires, Argentina

12 Departamento de Neurología, UA Cesar Milstein, Buenos Aires, Argentina

13 INECO Neurociencias Oroño - Fundación INECO, Rosario, Argentina

14 Servicio de Neurología, Hospital Provincial del Centenario, Rosario, Argentina

15 INEBA, Instituto neurociencias Buenos Aires, Buenos Aires, Argentina

16 Instituto de Neurociencias de Rosario, Rosario, Argentina

17 Department of Neuroscience - Neuroimmunology Unit, Hospital Alemán, Buenos Aires, Argentina

18 Multiple Sclerosis Section, Hospital Británico, Buenos Aires, Argentina

19 RIAPEM (Red Integral Asistencial al Paciente con Esclerosis Múltiple), Santiago del Estero, Argentina

20 Servicio de Neurología, Hospital Cárlos G. Durand, Buenos Aires, Argentina

21 Hospital San Martín, Paraná, Entre Ríos, Argentina

22 Departamento de Nuerología, Fundación Favaloro/INECO, Buenos Aires, Argentina 